Duckham, A. N. (1967). Fodder Conservation Occasional Symposium no. 3, p. 1. British Grassland Society.

Johansson, V. (r969). Agricultural College of Sweden Department of Economics (mimeograph).

Street, P. R. (x 969$)$. Discussion paper for the Agricultural Economics Society, July r 969 (mimeograph).

Thompson, S. C. (1967). Study Reading University Department of Agriculture no. 3.

Thompson, S. C. (1969). Farm \& Country, September.

\title{
Logging data in digital form
}

By S. W. R. Cox, Instrumentation Department, National Institute of Agricultural Engineering, Silsoe, Bedfordshire

This paper is not written from any special knowledge of the data-logging requirements of nutrition experiments, but from some experience in assisting fellow scientists and engineers in logging and processing their 'data'. Powerful aid can be provided by digital computers, backed by data-logging equipment, but in individual cases the benefits have to be balanced against both the cost of automatic logging and the time required to programme a computer for the subsequent data processing Further deterrents may be unfamiliarity with the various forms of data recording equipment that are available commercially and uncertainty about their relative usefulness for a given experiment, for there is continuing advance in the performance of equipment and in the development of digital computers. In the latter there is a trend towards greater cost and power on the one hand, and towards smaller size, lower cost and increased portability on the other. Smaller computers, integral with data-logging units, may remove the convenient division between the logging and the processing stages of data handling.

Any survey of the cost or performance of data-logging techniques is liable to be out of date in a year or two, but certain types of equipment are now being produced commercially which will probably provide the basis of logging systems for some time to come. Statements about the spheres of application of such equipment are therefore of value.

Before attempting such a survey of data loggers the reasons for choosing digital equipment should be considered, for the stages of development at N.I.A.E. over the past few years (Matthews, 1967,1969 ) are probably relevant to aims of many other experimental establishments.

\section{Analogue measurements}

Until a few years ago most recorded measurements of N.I.A.E. were in analogue form on $\mathrm{Y}$ - $\mathrm{T}$ chart (i.e. with the $\mathrm{X}$-axis representing time, $\mathrm{T}$ ). The exceptions were normally those relating to counting systems where the output is normally in pulse or digital form. The single or multi-point chart recorder was the most widely used device. Its accuracy and speed of response suit many applications and, even in the multi-point form, with its discontinuous traces, it often provides a convenient visual presentation of the results, 
Following the introduction of the ultraviolet light recorder, many uses for this instrument were found at N.I.A.E.; not just for recording signals fluctuating at frequencies in the $\mathrm{kHz}$ region (where the u.v. recorder has little competition) but in application where the potentiometric recorder would have been adequate in sensitivity and speed of response. The reasons for this are the flexibility inherent in optical recording of fluctuating signals and of $\mathrm{X}$ and $\mathrm{Y}$ datum lines. These enable the experimenter to adjust his $\mathrm{Y}$ base-lines for the most convenient separation or juxtaposition of the signal traces, and to choose suitably spaced time-interval markers on the X-axis. The more comprehensive models, which have control of 'shotlength' (a valuable feature at high chart speeds) and other facilities for tailoring the chart record to the immediate need, are extremely valuable general-purpose instruments. Those with incandescent lamps, capable of working from low-voltage batteries, make the galvanometer recorder suitable as much for field use as for the laboratory.

Although less widely used, the X-Y recorders can be valuable for presentation of the relationship between two variables over a chosen range. They have a fast response for an electro-mechanical writing system (up to $100 \mathrm{~cm} \mathrm{~s}^{-1}$ in some cases) and they have been used at N.I.A.E. in instrumentation and analogue computing systems.

Connexion of measurement sensors or transducers to these chart recorders is normally direct or via a signal conditioning stage, which amplifies or otherwise processes the transducer output to the requirement of the recorder. However, for some purposes it is necessary or more convenient to record the transducer signals on magnetic tape. Analogue tape recorders may be of the direct recording (d.r.) type, i.e. employing the same principle as the domestic tape recorder but with a greater uniformity of response over their working frequency range, or they may be of the frequency-modulated (f.m.) type. In the latter, the amplitude variations of the signal are converted into proportional frequency deviations from a central 'carrier' frequency generated by a high-frequency oscillator within the recorder. The resulting signal must be 'demodulated' on play-back into an analogue output device. The f.m. system, unlike the d.r. system, can be used with very slowly varying or steady direct current signals, and the multi-channel f.m. recorder is, in consequence, far more widely used in instrumentation systems. The particular value of intermediate tape-recording of the signal is that on play-back the signal can be filtered electrically before being applied to the chart recorder, so permitting various forms of frequency analysis of the signal. Flexibility is added by the ability to play back the recording at a speed different from that at which it was originally taken (step-up or step-down ratios as high as $16: 1$ can be obtained with some equipment). When lowfrequency phenomena have been recorded, speeding up on playback can considerably shorten analysis time. Formation of a continuous loop of tape selected from a recording allows automatic analysis, in combination with automatically scanned filter networks.

All these techniques still find application at N.I.A.E., because of their ability to provide clear, visual indication of the variations in measured quantities, in a fairly 
indestructible form, but the more advanced forms of signal analysis do require a considerable amount of costly, special-purpose equipment, together with experienced staff to operate it.

\section{Reduction of analogue records to digital form}

Disadvantages of the above methods increase with the amount of data recorded in analogue form, till a stage is reached where, unless staff is available, a backlog of uninterpreted material accumulates. At this stage, unless the experimenter is prepared to forgo much of the information he originally hoped to extract he must resort to chart reading equipment, for sampling at preset intervals along the $\mathrm{X}$-axis, and the ordinates must be converted into digital form and punched on paper-tape or typed out in decimal form. Automatic curve followers are commercially available, but in general are most reliable on clearly-defined, continuous, single traces, many traces being unsuitable for this equipment. The most widely used chart readers require the placing, over each point in turn, of a stylus or a lens viewer. On a pushbutton command from the operator the $\mathrm{X}$ and $\mathrm{Y}$ co-ordinates of the chosen point are recorded. Appropriate linear or non-linear scaling factors can be introduced on the Y-axis. The N.I.A.E. has used equipment of this type extensively, for over 2 years, on recorder chart and ciné film, and it has been found that an average conversion rate of one point $/ 2 \mathrm{~s}$ can be maintained by a trained operator for long periods (Matthews, I 969). However, this system requires equipment costing about $f_{2000}$ in its cheapest form, together with an operator who is willing and able to do a very monotonous task without losing concentration. Commercial chart-reading services are available and are more economic unless a chart reader unit can be kept at least $50 \%$ of full loading. Therefore, while the chart-reading facilities at N.I.A.E. will continue to be available, particularly for short runs on ciné and chart records taken during exploratory stages of experimental work, the trend at the Institute over the last 2 or 3 years has been towards digital-logging equipment once the pattern of measurement and analysis becomes clear. Digital equipment offers greater potential accuracy of measurement, greater ability to work from measurement sensors of all kinds, greater power of computer analysis, and the not so distant prospect of computer based supervision of the logging process itself.

\section{The elements of digital logging systems}

Specialized data-loggers have been made commercially and by many research establishments, including N.I.A.E. (Blight, r967; Budd \& Suffolk, r967; Constantine, 1967; Matthews, 1967), but the recent development of commercial loggers has been such that it is now both technically and economically satisfactory to base a data-logging system on one of them.

For analogue signals the logger must have an analogue-to-digital (a-d) conversion stage which samples the signal periodically and converts it into digital form. In most loggers a standard digital voltmeter (D.V.M.) is used. This incorporates an a-d stage; a range of sensitivities and a decimal display of the analogue voltage as well as the required digital output, which is usually in binary-coded decimal (B.C.D.) 
form. A good D.V.M. is little affected by the electrical interference radiated from neighbouring electrical appliances. This form of interference, which is picked up by the two input wires to the voltmeter, is termed 'common mode' and the voltmeter is said to have a high common mode rejection (C.M.R.) figure. It may also incorporate a filter to reduce any mains frequency component in the incoming analogue signal (series-mode rejection). The a-d stage will accept direct current or low frequency alternating current signals up to a given maximum voltage, frequently $100 \mathrm{mV}$. The output signal from the measurement transducer must therefore be conditioned to this level, and higher frequency components in the signal must be removed.

Digital input signals-whether from instrumentation or from a manual keyboard -will by-pass the a-d stage in the logger. Signals of this type must conform to the binary 'logic' levcls in the logger (e.g. $\circ$ and $-1 \circ \mathrm{V}$ ) and the type of binary coding employed.

Most loggers are designed for multi-channel operation, and contain a scanner stage immediately following the sensors and their signal conditioning circuits. All analogue inputs therefore time-share one a-d unit, through the common scanner stage, which usually incorporates sealed reed-switches for maximum reliability of switching, especially with low-level signals.

The coded digital output from each input data generator (analogue or digital) has to be passed to the chosen output storage device, which may be an electric typewriter or lister, a paper-tape punch or a digital (incremental) magnetic tape recorder. With the possible exception of the last-mentioned, it is first necessary to take the parallel-coded information and to serialize it. The serializer together with output circuits appropriate to the final recording device provides the latter with the measurement information (plus identification data) in the form of 'data words', suitably coded and spaced.

The complete logging system may also involve transmission of signals in analogue or digital form between widely separated points, either by wire or by radio-frequency links. R.f telemetry is sometimes the only means available to transmit signals from one stage of the logging system to the next. The technique is applied to transmission of signals from sensors mounted on or in freely moving bodies-men, animals or machines. Commercial multi-channel telemetry equipment for general industrial use is just beginning to obtain Post Office approval for application by licenced users. Although digital telemetry systems exist, this commercial equipment uses the f.m.-f.m. technique, i.e., frequency-modulation with frequency-multiplexing of the separate channels. The original signal is restored in analogue form at the output from the receiver. Where cable connexion can be made from remote measuring equipment to a central logging facility, cable runs up to several hundreds of metres can be accepted by suitably designed equipment. Analogue signals have been reliably conveyed in this way and over these distances in interference-prone environments. Coded digital data are accepted by Post Office lines for transmission to central logging and computing facilities. Hiring costs depend on the rate at which data will be transmitted. The Post Office 'Datel' service can offer rates of from 50 to 2400 bits (binary digits) per second, even if error detection is incorporated, and the public 
telephone network or a private line employed. The private line is less prone to generate spurious 'characters', for it has fewer switches and no time pips to act as false data.

Occasional errors will inevitably occur owing to malfunction of equipment. These must be deleted or circumvented by the computer to obviate loss of information. Considerable ingenuity may be needed in the programme writing to retain useful data while discarding or replacing erroneous characters (Rees, I967).

\section{Currently available commercial equipment}

Some very expensive and complex digital logging equipment is marketed, but here consideration is limited to more modest systems that are intended for wide use in experimental and testing work, i.e. systems costing up to about $£ 3500$.

An inexpensive hybrid system that can be useful if the measured quantities vary only slowly is obtainable. This is a standard potentiometric recorder, fitted with a coding disc coupled to the balancing motor, which gives a coded digital output representing the position of the recorder's pointer. The extra cost of the disc and decoding equipment is about $f_{0} 700-f_{900}$. The potentiometric recorder can also be fitted with a much cheaper device-the retransmitting slidewire-but this is only a means of generating a high-level analogue output from the recorder, proportional to the input signal, and this output must be put into an a-d device for digital logging.

Undoubtedly, the most versatile instruments now available are the d.v.m.-based loggers, of which there are several competing models priced at $f_{2000}$ to $\$ 35^{\circ}$. The basic system usually has a Io-channel capability, expandable up to roo channels, a maximum sensitivity of $100 \mathrm{mV}$ full range, flexible scanning programmes to allow single or repetitive scan of selected channels at one of a number of preset intervals, depending on the speed of the output device and also a range of options on the output recorder. Keyboards may be coupled in to add measurement or heading data manually. Some loggers have out-of-limits monitoring modules that cause an output record to be made when a measured value goes outside preset limits. The time of such an event can be logged by using a digital clock. For short-term use it is economic to hire these loggers.

It is now possible to expand an existing d.v.m. and an output device into a data logger by adding a unit alternatively known as a D.T.U. (data translation unit) or a coupler. This is, in effect, the serializer and drive unit for the output device in complete loggers, and is capable of accepting multiple BCD input signals. The cost varies with versatility from $f, 500$ to $f, 1000$.

Out-of-doors and in other difficult environments the digital magnetic tape logger may be best. A battery-powered instrument capable of ro-channel operation may cost

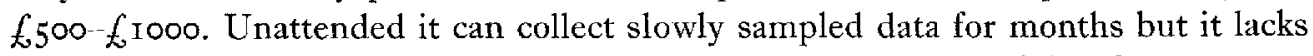
a visual display and needs translation facilities to convert the stored data for computer use. A ficld-test set with visual display costs about $f_{5} 500$ exclusive of the expensive translation equipment. It is usual to pay for the manufacturer's own translation service.

R.f. telemetry systems, with a range of a few hundred metres, typically cost about 
$£ 300$ per channel for up to 6 channels. A G.P.O. Datel system will cost upwards of $£_{2} 5^{\circ}$ per annum to hire.

\section{Some applications of data loggers}

In conclusion, two recent examples of data logging are described. (I) A standard d.v.m. logger has been set up to record on punched paper-tape weight change and temperature data from an N.I.A.E. drying installation. The installation contains eight automatically-balanced weigh-platforms, each carrying a seed-drying assembly. Heated air is passed into the individual driers through flexible pipes which do not affect the weight measurements appreciably. Weight loss on each drier is logged as the seeds lose moisture, by measuring the time taken for successive, pre-set weight decrements to occur. The punching rate is thus non-linear, like the drying curve. Heating and ambient air temperatures, together with rate of airflow are logged on a different time schedule, and punched separately. Separate feeding of the two sets of data into the computer reduces space requirements. (2) Measurement of the dimensions of vegetables has been speeded up at N.I.A.E. by the introduction of a d.v.m. logger, coupled to a callipers device made at N.I.A.E. (Kemp \& Perkins, 1970). The length or breadth of individual vegetables of different kinds can be recorded by the operation of a foot-switch, once the callipers have been set to the appropriate dimension. A keyboard is used to add identification data. With this equipment an operator can $\log$ dimensions at a rate of about $500 / \mathrm{h}$, with an accuracy of better than $\pm 0.5 \mathrm{~mm}$. A portable magnetic-tape logger and callipers for smaller fruit and vegetables, or for eggs, have been manufactured.

There is no doubt that manufacturers are interested in developing input units for their logging equipment, provided that they can see a modest market for these units. Instrumentation specialists foresee a great need for co-ordination of the requirements of experimenters who wish to record weight, dimensions, sample counts and environmental measurements.

\section{REFERENCES}

Blight, D. P. (1967). ARC Data Logging Symposium, National Institute of Agricultural Engineering (unpublished).

Budd, R. T. \& Suffolk, S. F. (1967). ARC Data Logging Symposium, National Institute of Agricultural Engineering (unpublished).

Constantine, T. R. ( 1967$)$. ARC Data Logging Symposium, National Institute of Agricultural Engineering (unpublished).

Kemp, D. C. \& Perkins, D. J. (1970). F. agric. Engng Res, 15, 75.

Matthews, J. (1967). ARC Data Logging Symposizm, National Institute of Agricultural Engineering (unpublished).

Matthews, J. (I969). Shelter Research Symposium, Ministry of Agriculture, Fisheries and Food (unpublished).

Rees, D. H. (I967). ARC Data Logging Symposium, National Institute of Agricultural Engineering (unpublished). 